\title{
Monotonicity, concavity, and inequalities related to the generalized digamma function
}

Li Yin ${ }^{1 *}$, Li-Guo Huang ${ }^{1}$, Xiu-Li Lin² and Yong-Li Wang ${ }^{3}$

"Correspondence: yinli_79@163.com

${ }^{1}$ College of Science, Binzhou University, Binzhou City, China Full list of author information is available at the end of the article

\begin{abstract}
In this paper, we establish a concave theorem and some inequalities for the generalized digamma function. Hence, we give complete monotonicity property of a determinant function involving all kinds of derivatives of the generalized digamma function.
\end{abstract}

MSC: 33B15

Keywords: Generalized digamma function; Complete monotonicity; Inequality; Concavity

\section{Introduction}

It is well known that the Euler gamma function is defined by

$$
\Gamma(x)=\int_{0}^{\infty} t^{x-1} e^{-t} d t, \quad x>0 .
$$

The logarithmic derivative of $\Gamma(x)$ is called the psi or digamma function. That is,

$$
\begin{aligned}
\psi(x) & =\frac{d}{d x} \ln \Gamma(x)=\frac{\Gamma^{\prime}(x)}{\Gamma(x)} \\
& =-\gamma-\frac{1}{x}+\sum_{n=1}^{\infty} \frac{x}{n(n+x)},
\end{aligned}
$$

where $\gamma=0.5772 \ldots$ is the Euler-Mascheroni constant. The gamma, digamma, and polygamma functions play an important role in the theory of special function, and have many applications in many other branches such as statistics, fractional differential equations, mathematical physics, and theory of infinite series. The reader may see related references $[5,7,9,13,16,23-28]$.

In [6], the $k$-analogue of the gamma function is defined for $k>0$ and $x>0$ as follows:

$$
\begin{aligned}
\Gamma_{k}(x) & =\int_{0}^{\infty} t^{x-1} e^{-\frac{t^{k}}{k}} d t \\
& =\lim _{n \rightarrow \infty} \frac{n ! k^{n}(n k)^{\frac{x}{k}-1}}{(x)_{n, k}},
\end{aligned}
$$

(c) The Author(s) 2018. This article is distributed under the terms of the Creative Commons Attribution 4.0 International License (http://creativecommons.org/licenses/by/4.0/), which permits unrestricted use, distribution, and reproduction in any medium, provided you give appropriate credit to the original author(s) and the source, provide a link to the Creative Commons license, and indicate if changes were made. 
where $\lim _{k \rightarrow 1} \Gamma_{k}(x)=\Gamma(x)$. It is natural that the $k$-analogue of the digamma function is defined for $x>0$ by

$$
\psi_{k}(x)=\frac{d}{d x} \log \Gamma_{k}(x)=\frac{\Gamma_{k}^{\prime}(x)}{\Gamma_{k}(x)} .
$$

It is worth noting that Nantomah et al. gave $(p, k)$-analogue of the gamma and the digamma functions in [15]. Further, they established some inequalities involving these new functions. The reader may see references $[14,15]$.

Very recently, Alzer and Jameson [2] presented a harmonic mean inequality for the digamma function, and also showed some interesting inequalities. It is natural to ask if one can generalize these results to the generalized digamma function with single parameters. This is the first object in this paper.

The second object of this paper came from the article of Ismail and Laforgia. In [11], they proved complete monotonicity of a determinant function involving the derivatives of the digamma function. Using their idea, we prove that their conclusion is also true for the generalized digamma function. In particular, some of the work about the complete monotonicity of these special functions may be found in $[3,4,8,10,12,17-22]$.

\section{Lemmas}

Lemma 2.1 For $k>0$ and $x>0$, the following identities hold true:

$$
\begin{aligned}
\Gamma_{k}(x) & =k^{\frac{x}{k}-1} \Gamma\left(\frac{x}{k}\right), \\
\psi_{k}(x) & =\frac{\ln k}{k}+\frac{1}{k} \psi\left(\frac{x}{k}\right) .
\end{aligned}
$$

Proof Using the substitution $\frac{t}{\sqrt[k]{k}}=u$ and $u^{k}=p$, we easily obtain

$$
\begin{aligned}
\Gamma_{k}(x) & =(\sqrt[k]{k})^{x} \int_{0}^{\infty}\left(\frac{t}{\sqrt[k]{k}}\right)^{x-1} e^{-\left(\frac{t}{\sqrt[k]{k}}\right)^{k}} d\left(\frac{t}{\sqrt[k]{k}}\right) \\
& =k^{\frac{x}{k}} \int_{0}^{\infty} u^{x-1} e^{-u^{k}} d u \\
& =k^{\frac{x}{k}-1} \int_{0}^{\infty} p^{\frac{x}{k}-1} e^{-p} d p \\
& =k^{\frac{x}{k}-1} \Gamma\left(\frac{x}{k}\right) .
\end{aligned}
$$

So, we prove formula (2.1). To (2.2), direct computation yields

$$
\begin{aligned}
\psi_{k}(x) & =\frac{\Gamma_{k}^{\prime}(x)}{\Gamma_{k}(x)}=\frac{\left[k^{\frac{x}{k}-1} \Gamma\left(\frac{x}{k}\right)\right]^{\prime}}{\left[k^{\frac{x}{k}-1} \Gamma\left(\frac{x}{k}\right)\right]} \\
& =\frac{\ln k}{k}+\frac{1}{k} \psi\left(\frac{x}{k}\right) .
\end{aligned}
$$

Lemma $2.2([1,2])$ For $x>0$, we have

$$
\psi^{\prime}(x)<\frac{1}{x}+\frac{1}{2 x^{2}}+\frac{1}{6 x^{3}}
$$


and

$$
\psi^{\prime \prime}(x)<-\frac{1}{x^{2}}-\frac{1}{x^{3}}
$$

Lemma 2.3 For $k, x>0, m \in \mathbb{N}$, we have

$$
\psi_{k}^{(m)}(x)=(-1)^{m+1} m ! \sum_{n=0}^{\infty} \frac{1}{(n k+x)^{m+1}}
$$

and

$$
\psi_{k}^{(m)}(x)=(-1)^{m+1} \int_{0}^{\infty} \frac{t^{m}}{1-e^{-k t}} e^{-x t} d t .
$$

Proof Formula (2.5) may be found in reference [15]. By using formula (9) in reference [15], we have

$$
\begin{aligned}
\psi_{k}^{(m)}(x) & =\lim _{p \rightarrow \infty} \psi_{p, k}^{(m)}(x) \\
& =\lim _{p \rightarrow \infty}(-1)^{m+1} \int_{0}^{\infty}\left(\frac{1-e^{-k(p+1) t}}{1-e^{-k t}}\right) t^{m} e^{-x t} d t \\
& =(-1)^{m+1} \int_{0}^{\infty} \frac{1}{1-e^{-k t}} t^{m} e^{-x t} d t
\end{aligned}
$$

So, we prove formula (2.6).

\section{Main results}

Theorem 3.1 For $k>0$, the function $x^{2} \psi_{k}^{\prime}(x)$ is strictly increasing on $(0, \infty)$.

Proof Using Lemma 2.1, we have

$$
\psi_{k}^{\prime}(x)=\frac{1}{k^{2}} \psi^{\prime}\left(\frac{x}{k}\right) \quad \text { and } \quad \psi_{k}^{\prime \prime}(x)=\frac{1}{k^{3}} \psi^{\prime \prime}\left(\frac{x}{k}\right)
$$

Combining with the identity $\psi^{(m)}(x)=(-1)^{m+1} m ! \sum_{n=0}^{\infty} \frac{1}{(n+x)^{m+1}}$, we get

$$
\begin{aligned}
\frac{d}{d x}\left(x^{2} \psi_{k}^{\prime}(x)\right) & =\frac{2 x}{k^{2}} \psi^{\prime}\left(\frac{x}{k}\right)+\frac{x^{2}}{k^{3}} \psi^{\prime \prime}\left(\frac{x}{k}\right) \\
& =2 x \sum_{n=0}^{\infty} \frac{n k}{(n k+x)^{3}}>0 .
\end{aligned}
$$

Theorem 3.2 For $k>0$, the function $\psi_{k}\left(\frac{1}{x}\right)$ is strictly concave on $(0, \infty)$.

Proof Easy computation results in

$$
\frac{d}{d x}\left(\psi_{k}\left(\frac{1}{x}\right)\right)=-\frac{1}{x^{2}} \psi_{k}^{\prime}\left(\frac{1}{x}\right) .
$$

Considering Theorem 3.1, we complete the proof. 
Theorem 3.3 For $k \geq \frac{1}{\sqrt[3]{3}}=0.693361 \ldots$, the function

$$
\lambda_{k}(x)=\psi_{k}(x)+\psi_{k}\left(\frac{1}{x}\right)
$$

is strictly concave on $(0, \infty)$.

Proof By differentiation and applying Lemma 2.1, we easily obtain

$$
\begin{aligned}
& \lambda_{k}^{\prime}(x)=\psi_{k}^{\prime}(x)-\frac{1}{x^{2}} \psi_{k}^{\prime}\left(\frac{1}{x}\right), \\
& \lambda_{k}^{\prime \prime}(x)=\psi_{k}^{\prime \prime}(x)+\frac{2}{x^{3}} \psi_{k}^{\prime}\left(\frac{1}{x}\right)+\frac{1}{x^{4}} \psi_{k}^{\prime \prime}\left(\frac{1}{x}\right),
\end{aligned}
$$

and

$$
k^{3} x^{4} \lambda_{k}^{\prime \prime}(x)=x^{4} \psi^{\prime \prime}\left(\frac{x}{k}\right)+2 k x \psi^{\prime}\left(\frac{1}{k x}\right)+\psi^{\prime \prime}\left(\frac{1}{k x}\right) .
$$

Applying Lemma $2.2, k \geq \frac{1}{\sqrt[3]{3}}$, and the recurrence relations

$$
\begin{aligned}
& \psi^{\prime}\left(\frac{1}{k x}+1\right)=\psi^{\prime}\left(\frac{1}{k x}\right)-k^{2} x^{2}, \\
& \psi^{\prime \prime}\left(\frac{1}{k x}+1\right)=\psi^{\prime \prime}\left(\frac{1}{k x}\right)+2 k^{3} x^{3},
\end{aligned}
$$

we have

$$
\begin{aligned}
k^{3} x^{4} \lambda_{k}^{\prime \prime}(x)= & x^{4} \psi^{\prime \prime}\left(\frac{x}{k}\right)+2 k x \psi^{\prime}\left(\frac{1}{k x}\right)+\psi^{\prime \prime}\left(\frac{1}{k x}\right) \\
< & x^{4}\left(-\frac{k^{2}}{x^{2}}-\frac{k^{3}}{x^{3}}\right)+2 k x\left[\frac{k x}{1+k x}+\frac{k^{2} x^{2}}{2(1+k x)^{2}}+\frac{k^{3} x^{3}}{6(1+k x)^{3}}\right] \\
& -\frac{k^{2} x^{2}}{(1+k x)^{2}}-\frac{k^{3} x^{3}}{(1+k x)^{3}} \\
= & -\frac{k x}{3(1+k x)^{3}}\left[3 k^{2}+9 k^{3} x+9 k^{2} x^{2}+k^{2}\left(3 k^{3}-1\right) x^{3}+3 k^{4} x^{4}\right] \\
< & 0 .
\end{aligned}
$$

This implies that $\lambda_{k}(x)$ is strictly concave on $(0, \infty)$.

Theorem 3.4 For $x \in(0, \infty)$ and $k \geq \frac{1}{\sqrt[3]{3}}$, we have

$$
\psi_{k}(x)+\psi_{k}\left(\frac{1}{x}\right) \leq \frac{2 \ln k+2 \psi\left(\frac{1}{k}\right)}{k}
$$

Proof Since the function $\lambda_{k}(x)=\psi_{k}(x)+\psi_{k}\left(\frac{1}{x}\right)$ is strictly concave on $(0, \infty)$, we get

$$
\lambda_{k}^{\prime}(x) \geq \lambda_{k}^{\prime}(1)=0, \quad x \in(0,1]
$$


and

$$
\lambda_{k}^{\prime}(x) \leq \lambda_{k}^{\prime}(1)=0, \quad x \in[1, \infty)
$$

It follows that $\lambda_{k}$ is increasing on $(0,1]$ and decreasing on $[1, \infty)$. Hence, $\lambda_{k}(x) \leq \lambda_{k}(1)$ for $x>0$. The proof is complete.

Remark 3.1 Let $\gamma_{k}=-\psi_{k}(1)=-\frac{\ln k}{k}-\frac{1}{k} \psi\left(\frac{1}{k}\right)$ be the $k$-analogue of the Euler-Mascheroni constant. It is obvious that $\lim _{k \rightarrow 1} \gamma_{k}=\gamma$.

Definition 3.1 It is known that the generalized digamma function $\psi_{k}(x)$ is strictly increasing on $(0, \infty)$ with $\psi_{k}\left(0^{+}\right) \psi_{k}(\infty)<0$. So, the function has a sole positive root in $(0, \infty)$. We define this positive root for $x_{k}$. That is,

$$
\ln k+\psi\left(\frac{x_{k}}{k}\right)=0
$$

Theorem 3.5 For $x \in(0,1)$ and $\frac{1}{\sqrt[3]{3}} \leq k \leq 1$, we have

$$
\psi_{k}(1+x) \psi_{k}(1-x) \leq \frac{\ln ^{2} k+\gamma^{2}-2(\gamma+1) \ln k}{k^{2}}
$$

Proof Considering $\frac{1}{\sqrt[3]{3}} \leq k \leq 1$ and the definition of $x_{k}$, we have

$$
\frac{1}{\sqrt[3]{3}} x_{0} \leq x_{k} \leq x_{0}
$$

where $x_{0}$ satisfies $\psi\left(x_{0}\right)=0$ with $x_{0}=1.46163 \ldots$

Case 1. If $x \in\left[x_{k}-1,1\right)$, then we have $\psi_{k}(1-x) \leq 0 \leq \psi_{k}(1+x)$. This implies that formula (3.2) holds.

Case 2. If $x \in\left(0, x_{k}-1\right]$, using the power series expansion

$$
\psi(1+z)=-\gamma+\sum_{k=2}^{\infty}(-1)^{k} \zeta(k) z^{k-1}, \quad|z|<1,
$$

we obtain

$$
\begin{aligned}
\psi_{k}(1+x) & \geq \psi_{k}(k+x)=\frac{\ln k}{k}+\frac{1}{k} \psi\left(1+\frac{x}{k}\right) \\
& =\frac{\ln k}{k}+\frac{1}{k}\left[-\gamma+\sum_{k=2}^{\infty}(-1)^{k} \zeta(k) x^{k-1}\right],
\end{aligned}
$$

where $\zeta(k)=\sum_{n=1}^{\infty} \frac{1}{n^{k}}$ is the Riemann zeta function.

Furthermore, we have

$$
0<-\psi_{k}(1+x) \leq-\frac{\ln k}{k}+\frac{1}{k}\left[\gamma-\zeta(2) x+\zeta(3) x^{2}\right] .
$$


Completely similar to (3.4), we have

$$
\begin{aligned}
0 & <-\psi_{k}(1-x) \leq-\frac{\ln k}{k}+\frac{1}{k}\left[\gamma+\zeta(2) y+\zeta(3) \sum_{k=2}^{\infty} x^{k}\right] \\
& \leq-\frac{\ln k}{k}+\frac{1}{k}\left[\gamma+\zeta(2) x+\zeta(3) x^{2}\right] .
\end{aligned}
$$

Combining (3.4) with (3.5), we obtain

$$
\psi_{k}(1+x) \psi_{k}(1-x) \leq \frac{\ln ^{2} k+\gamma^{2}-2(\gamma+1) \ln k}{k^{2}}
$$

by using $\zeta(3) x^{2}<1$.

Theorem 3.6 For $x \in(0, \infty)$ and $\frac{1}{\sqrt[3]{3}} \leq k \leq 1$, we have

$$
\psi_{k}(x) \cdot \psi_{k}\left(\frac{1}{x}\right) \leq \frac{\ln ^{2} k+\gamma^{2}-2(\gamma+1) \ln k}{k^{2}} .
$$

Proof We only need to prove (3.6) for $x \geq 1$. If $x \geq x_{k}$, then we get $\psi_{k}\left(\frac{1}{x}\right) \leq 0 \leq \psi_{k}(x)$. It follows that inequality (3.6) holds true.

If $x \in\left(1, x_{k}\right]$ and setting $x=1+z$, we get

$$
\psi_{k}(1-z) \leq \psi_{k}\left(\frac{1}{x}\right)
$$

Therefore, we have

$$
\begin{aligned}
\psi_{k}(x) \cdot \psi_{k}\left(\frac{1}{x}\right) & =\psi_{k}(1+z) \psi_{k}\left(\frac{1}{x}\right) \\
& \leq \psi_{k}(1+z) \psi_{k}(1-z) \\
& \leq \frac{\ln ^{2} k+\gamma^{2}-2(\gamma+1) \ln k}{k^{2}}
\end{aligned}
$$

by using Theorem 3.5 .

Corollary 3.1 For $x \in(0, \infty)$ and $\frac{1}{\sqrt[3]{3}} \leq k \leq 1$, we have

$$
\frac{2 \psi_{k}(x) \psi_{k}\left(\frac{1}{x}\right)}{\psi_{k}(x)+\psi_{k}\left(\frac{1}{x}\right)} \geq \frac{\ln ^{2} k+\gamma^{2}-2(\gamma+1) \ln k}{k\left[\ln k+\psi\left(\frac{1}{k}\right)\right]} .
$$

Proof Applying Theorems 3.4 and 3.6, we obtain

$$
\begin{aligned}
\frac{2 \psi_{k}(x) \psi_{k}\left(\frac{1}{x}\right)}{\psi_{k}(x)+\psi_{k}\left(\frac{1}{x}\right)} & \geq 2 \cdot \frac{\ln ^{2} k+\gamma^{2}-2(\gamma+1) \ln k}{k^{2}} \frac{1}{\psi_{k}(x)+\psi_{k}\left(\frac{1}{x}\right)} \\
& \geq \frac{\ln ^{2} k+\gamma^{2}-2(\gamma+1) \ln k}{k^{2}} \frac{k}{\ln k+\psi\left(\frac{1}{k}\right)} .
\end{aligned}
$$

The proof is complete. 
Next, for $m, n, j \in \mathbb{N}$, we define the function $\mu_{n}$ by

$$
\mu_{n}(x)=\left|\begin{array}{cccc}
\psi_{k}^{(m)}(x) & \psi_{k}^{(m+j)}(x) & \cdots & \psi_{k}^{(m+n j)}(x) \\
\psi_{k}^{(m+j)}(x) & \psi_{k}^{(m+2 j)}(x) & \cdots & \psi_{k}^{[m+(n+1) j]}(x) \\
\vdots & \vdots & & \vdots \\
\psi_{k}^{(m+n j)}(x) & \psi_{k}^{(m+(n+1) j)}(x) & \cdots & \psi_{k}^{(m+2 n j)}(x)
\end{array}\right|
$$

Completely similar to the method in [11], the following Theorem 3.7 can be proved.

Theorem 3.7 For $m, n, j \in \mathbb{N}$, then $(-1)^{(n+1)(m+1)} \mu_{n}(x)$ is completely monotonic on $(0, \infty)$.

Proof Using Lemma 2.3, we have

$$
\begin{aligned}
& \mu_{n}(x)=(-1)^{n+1} \underbrace{\int_{-\infty}^{0} \cdots \int_{-\infty}^{0}}_{n+1 \text { times }}\left|\begin{array}{cccc}
u_{0}^{m} & u_{0}^{m+j} & \cdots & u_{0}^{m+n j} \\
u_{1}^{m+j} & u_{1}^{m+2 j} & \cdots & u_{1}^{m+(n+1) j} \\
\vdots & \vdots & & \vdots \\
u_{n}^{m+n j} & u_{n}^{m+(n+1) j} & \cdots & u_{n}^{m+2 n j}
\end{array}\right| \\
& \cdot \frac{e^{\frac{x}{k}\left(u_{0}+u_{1}+\cdots+u_{n}\right)}}{\prod_{i=0}^{n}\left(1-e^{u_{i}}\right)} d u_{0} d u_{1} \cdots d u_{n} \\
& =(-1)^{n+1} \underbrace{\int_{-\infty}^{0} \cdots \int_{-\infty}^{0}}_{n+1 \text { times }}\left|\begin{array}{cccc}
u_{\delta(0)}^{m} & u_{\delta(0)}^{m+j} & \cdots & u_{\delta(0)}^{m+n j} \\
u_{\delta(1)}^{m+j} & u_{\delta(1)}^{m+2 j} & \cdots & u_{\delta(1)}^{m+(n+1) j} \\
\vdots & \vdots & & \vdots \\
u_{\delta(n)}^{m+n j} & u_{\delta(n)}^{m+(n+1) j} & \cdots & u_{\delta(n)}^{m+2 n j}
\end{array}\right| \\
& \cdot \frac{e^{\frac{x}{k}\left(u_{0}+u_{1}+\cdots+u_{n}\right)}}{\prod_{i=0}^{n}\left(1-e^{u_{i}}\right)} d u_{0} d u_{1} \cdots d u_{n} \text {, }
\end{aligned}
$$

where $\delta$ is a permutation on $0,1,2, \ldots, n$.

Let $\operatorname{sgn}(\delta)$ be the sign of $\delta$, we can obtain

$$
\begin{aligned}
& u_{n}(x)=(-1)^{n+1} \underbrace{\int_{-\infty}^{0} \cdots \int_{-\infty}^{0}}_{n+1 \text { times }} \frac{e^{\frac{x}{k}\left(u_{0}+u_{1}+\cdots+u_{n}\right)}}{\prod_{i=0}^{n}\left(1-e^{u_{i}}\right)} \operatorname{sgn}(\delta) \prod_{i=0}^{n} u_{i}^{m} \\
& \left|\begin{array}{cccc}
u_{0}^{0} & u_{0}^{j} & \cdots & u_{0}^{n j} \\
u_{1}^{j} & u_{1}^{2 j} & \cdots & u_{1}^{(n+1) j} \\
\vdots & \vdots & & \vdots \\
u_{n}^{n j} & u_{n}^{(n+1) j} & \ldots & u_{n}^{2 n j}
\end{array}\right| d u_{0} d u_{1} \cdots d u_{n}
\end{aligned}
$$

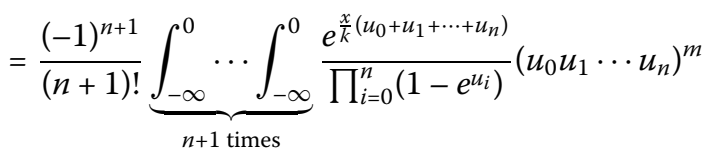

$$
\begin{aligned}
& \cdot \prod_{0 \leq i<l \leq n}\left(u_{i}^{j}-u_{l}^{j}\right) d u_{0} d u_{1} \cdots d u_{n}
\end{aligned}
$$


Replacing $u_{0}, u_{1}, \ldots, u_{n}$ by $-u_{0},-u_{1}, \ldots,-u_{n}$, we get

$$
\begin{aligned}
\mu_{n}(x)= & (-1)^{(n+1)(m+1)} \underbrace{\int_{0}^{\infty} \cdots \int_{0}^{\infty} e^{-\frac{x}{k}\left(u_{0}+u_{1}+\cdots+u_{n}\right)}}_{n+1 \text { times }} \\
& \cdot \prod_{0 \leq i<l \leq n}\left(u_{i}^{j}-u_{l}^{j}\right) \prod_{i=0}^{n} \frac{u_{i}^{n}}{1-e^{-u_{i}}} d u_{0} d u_{1} \cdots d u_{n} .
\end{aligned}
$$

This implies that $(-1)^{(n+1)(m+1)} \mu_{n}(x)$ is completely monotonic.

By taking $n=1$, the following Corollary 3.2 can be easily obtained.

Corollary 3.2 For $m, j \in \mathbb{N}$, and $x>0$, we have

$$
\left|\begin{array}{cc}
\psi_{k}^{(m)}(x) & \psi_{k}^{(m+j)}(x) \\
\psi_{k}^{(m+j)}(x) & \psi_{k}^{(m+2 j)}(x)
\end{array}\right|>0 .
$$

\section{Conclusions}

We established a concave theorem and some monotonic properties for the generalized digamma function, and some interesting inequalities were obtained. These conclusions generalize Alzer's results. On the other hand, we prove a completely monotonic property for the generalized digamma function by using Ismail and Laforgia's idea.

\section{Methods and experiment}

Not applicable.

\section{Acknowledgements}

The authors would like to thank the editor and the anonymous referee for their valuable suggestions and comments, which helped us to improve this paper greatly.

\section{Funding}

The authors were supported by the National Natural Science Foundation of China (Grant Nos. 11401041, 11705122), the Science and Technology Foundations of Shandong Province (Grant Nos. J16li52 and J14li54) and Science Foundations of Binzhou University (Grant Nos. BZXYL1104 and BZXYL1704).

\section{Competing interests}

The authors declare that they have no competing interests.

\section{Authors' contributions}

All authors contributed equally to the manuscript and read and approved the final manuscript.

\section{Author details}

${ }^{1}$ College of Science, Binzhou University, Binzhou City, China. ${ }^{2}$ College of Mathematics Science, Qufu Normal University, Qufu City, China. ${ }^{3}$ College of Mathematics and Systems Science, Shandong University of Science and Technology, Qingdao, China.

\section{Publisher's Note}

Springer Nature remains neutral with regard to jurisdictional claims in published maps and institutional affiliations.

Received: 19 February 2018 Accepted: 2 July 2018 Published online: 20 July 2018

\section{References}

1. Alzer, H.: On some inequalities for the gamma and psi functions. Math. Comput. 66, 373-389 (1997)

2. Alzer, H., Jameson, G.: A harmonic mean inequality for the digamma function and related results. Rend. Semin. Mat. Univ. Padova 137, 203-209 (2017) 
3. Berg, C., Pedersen, H.L.: A completely monotone function related to the gamma function. J. Comput. Appl. Math. 133, 219-230 (2001)

4. Chiu, S.N., Yin, C.-C.: On the complete monotonicity of the compound geometric convolution with applications to risk theory. Scand. Actuar. J. 2014(2), 116-124 (2014)

5. Coffey, M.C.: On one-dimensional digamma and polygamma series related to the evaluation of Feynman diagrams. J. Comput. Appl. Math. 183, 84-100 (2005)

6. Diaz, R., Pariguan, E.: On hypergeometric functions and Pochhammer k-symbol. Divulg. Math. 15(2), 179-192 (2007)

7. Doelder, P.J.: On some series containing $\psi(x)-\psi(y)$ and $(\psi(x)-\psi(y))^{2}$ for certain values of $x$ and $y$. J. Comput. Appl. Math. 37, 125-141 (1991)

8. Dong, H., Yin, C.-C.: Complete monotonicity of the probability of ruin and DE Finetti's dividend problem. J. Syst. Sci. Complex. 25(1), 178-185 (2012)

9. Feng, Q., Meng, F.-W.: Some new Gronwall-type inequalities arising in the research of fractional differential equations. J. Inequal. Appl. 2013, 429 (2013)

10. Grinshpan, A.Z., Ismail, M.E.H.: Completely monotonic functions involving the gamma and q-functions. Proc. Am. Math. Soc. 134, 1153-1160 (2006)

11. Ismail, M.E.H., Laforgia, A.: Monotonicity properties of determinants of special functions. Constr. Approx. 26, 1-9 (2007)

12. Ismail, M.E.H., Muldoon, M.E., Lorch, L.: Completely monotonic functions associated with the gamma function and its q-analogues. J. Math. Anal. Appl. 116, 1-9 (1986)

13. Murty, M.R., Saradha, N.: Transcendental values of the digamma function. J. Number Theory 125, 298-318 (2007)

14. Nantomah, K.: Convexity properties and inequalities concerning the $(p, k)$-gamma function. Commun. Fac. Sci. Univ. Ank. Sér. A1 66(2), 130-140 (2017)

15. Nantomah, K., Prempeh, E., Twum, S.B.: On a $(p, k)$-analogue of the gamma function and some associated inequalities. Moroccan J. Pure Appl. Anal. 2(2), 79-90 (2016)

16. Ogreid, O.M., Osland, P.: Some infinite series related to Feynman diagrams. J. Comput. Appl. Math. 140,659-671 (2002)

17. Qi, F., Chen, C.P.: A complete monotonicity property of the gamma function. J. Math. Anal. Appl. 296, 603-607 (2004)

18. Qi, F., Guo, B.-N.: Complete monotonicities of functions involving the gamma and digamma functions. RGMIA Res. Rep. Collect. 7(1), 63-72 (2004)

19. Qi, F., Guo, B.-N.: Some logarithmically completely monotonic functions related to the gamma function. J. Korean Math. Soc. 47(6), 1283-1297 (2010)

20. Qi, F., Guo, B.-N., Chen, C.-P.: Some completely monotonic functions involving the gamma and polygamma functions. RGMIA Res. Rep. Collect. 7(1), 31-36 (2004)

21. Qi, F., Guo, B.-N., Chen, C.-P.: Some completely monotonic functions involving the gamma and polygamma functions. J. Aust. Math. Soc. 80, 81-88 (2006)

22. Qi, F., Guo, S.-L., Guo, B.-N.: Complete monotonicity of some functions involving polygamma functions. J. Comput. Appl. Math. 233, 2149-2160 (2010)

23. Shao, J., Meng, F.-W.: Gronwall-Bellman type inequalities and their applications to fractional differential equations. Abstr. Appl. Anal. 2013, Article ID 217641 (2013)

24. Wang, Y., Liu, L.-S., Wu, Y.-H.: Positive solutions for a nonlocal fractional differential equation. Nonlinear Anal. 74(11), 3599-3605 (2011)

25. Wang, Y., Liu, L.-S., Wu, Y.-H.: Existence and uniqueness of a positive solution to singular fractional differential equations. Bound. Value Probl. 2012, 81 (2012)

26. Wang, Y., Liu, L.-S., Wu, Y.-H.: Positive solutions for a class of higher-order singular semipositone fractional differential system. Adv. Differ. Equ. 2014, 268 (2014)

27. Xu, R., Meng, F.-W.: Some new weakly singular integral inequalities and their applications to fractional differential equations. J. Inequal. Appl. 2016, 78 (2016)

28. Zheng, Z.-W., Zhang, X.-J., Shao, J.: Existence for certain systems of nonlinear fractional differential equations. J. Appl. Math. 2014, Article ID 376924 (2014)

\section{Submit your manuscript to a SpringerOpen ${ }^{\circ}$ journal and benefit from:}

- Convenient online submission

- Rigorous peer review

- Open access: articles freely available online

- High visibility within the field

- Retaining the copyright to your article

Submit your next manuscript at $\gg$ springeropen.com 